\title{
Un exemple de calcul précis de la période optima de remplacement d'un matériel
}

\author{
Remplacement des roues de turbines dans une installation hydroélectrique

\section{Calculating the most economical working life of a hydraulic turbine runner}

\author{
PAR M. DANIEL
}

\begin{abstract}
INGENIEUR EN CONSTRUGTIONS GIVLLES DIPLOMÉ PAR L'ǴTAT
AUDTTEUR AU CONSERVATOIRE NaTIONAI DES ARTS ET MÉTIEHS

CHAIRE ET LABORATOIRE D'ÉCONOMIE INDUSTRIELLE ET STATISTIQUE
\end{abstract}

\begin{abstract}
L'usure des roues de turbines hydranliques abaisse notablement leur rendement: il en résulte soit des pertes d'énergie, soit un gaspillage d'eau. Leur remplacement se traduit par des frais et des pertes d'énergie, - Calcul de la période optimum de remplacement, dans le cas où l'eau est surabondante, puis dans celui où elle doit étre économisée. - Exemple de caicul pratique de la période optimum; estimation du préjudice causé par une erreur d'appréciation.
\end{abstract}

Dans son cours d'Hydraulique générale de 1942-43, M. Erdoux, professeur à l'Ecole Nationale des Ponts et Chaussées, aprés avoir montré la perte annuelle considérable qui peut résulter de l'emploi d'une roue de turbine ayant un rendement de $70 \%$, au lieu d'une roue à rendement de $85 \%$, s'exprime comme suit :

"D'autre part, les roues peuvent, dans certains cas, s'user très vite, parfois en 400 jours, et le rendement diminue rapidement avec l'usure. Or, pour le changement d'une roue, il faut compter, en plus de la perte correspondant à un jour perdu, une somme de 30 à $50.000 \mathrm{~F}$ pour des turbines moyennes, parfaitement négligeable au regard de la perte qu'occasionnerait une

\begin{abstract}
Wear of hydraulic turbine runners greatly reduces their efficiency, either from losses of energy or from losses of water, Replacing them involves expense and losses of energy. Calculation of the optimum period for replacement in the two cases where water is either superabundant or must be conserved. Example of practical calculation; estimate of losses caused by an error in calcnlating the optimum period before replacement.
\end{abstract}

marche prolongè à faible rendement par suite de l'usure de la roue.

" Pour toutes ces raisons, on voit qu'il faul faire des mesures sérieuses de rendement au début pour être sûr d'obtenir les chifrres de garantie et qu'en outre il faut faire des vérifications fréquentes du rendement en cours d'exploitation; il est intéressant de les faire avec le maximum de précision possible, surtout en ce qui concerne les essais de réception, car un gain de $1 \%$ sur le rendement entraine dans l'exemple précédent un bénéfice annuel de $75.000 \mathrm{~F}$. 》

A la lecture de ce passage, l'idée nous est venue de faire un calcul économique pour dé- 
Leminer rationnellement la période optima de remplacement d'une roue de turbine pour cause d'usure. Ce calcul peut avoir en effet un grand intérèt pour les roues s'usant rapidement : roues Pelton (hautes chules) ou roues-hélices (basses chutes) et dans les cas d'eau chargée. Dans les autres cas, la question n'est pent-être jamais négligeable.

Le vieillissement de la turbine dù à l'usure de celle-ci n'a pas les mêmes conséquences dans les usines an fil de l'eau et dans les usines à réservoir.

Dans le premicr cas, l'eau non turbinéc an passage est perdue par déversement. Il s'argit alors, pour chaque turbine en service, d'utiliser atu maximum l'eau qui lui est offerte à chaque instant par le cours d'eau. La baisse de production electrique est seule en cause, tout à fail indépendamment de la consommation d'eau, celle-ci étant gratuite.

Dans le second cas (réservoir à niveau variable), l'eau non utilisée par la turbine demeure stockće dans le réservoir où elle représente une énergie disponible. Le débit du réservoir est réglé rationnellement et, en première approximation, indépendamment de l'usure des turbines, par modification du nombre des turbines en service; d'autre part, le débit de chaque turbine en service est réglé, par mancuvre automatique du distributeur et quelquefois des aubes, de manière à donner l'optimum, c'est-à-dire ici le maximum de rendement hydraulique. Le volume d'eau par kWh produit, consommé en plus, du fait de l'usture, correspond done à une perte d'energie due uniquement à la perte de rendement technique. La distinction entre les deux cas n'est d'ailleurs pas si nette quon pourrait le croire à la lecture de ce qui précède. Certaines usines au fil de l'eau peuvent en efret, en période d'étiage, travailler à hauteur de chute variable, le barrage mobile étant complètement fermé. Cette réflexion nous conduit à distinguer, ici, au lieu des deux cas concrets du barrage réservoir ct de l'usine au fil de l'eau, les deux cas théoriques suivants :

A : L'eau est surabondante. Elle ne représente pas une énergie à ćconomiser;

B : L'eau esl stockée. Elle représente une énergic à économiser pour les besoins futurs.

Nous remarquerons en passant que la technique de la construction des turbines, tout orientíe par la notion du rendement maximum, semble être faile uniquement pour le second cas. On pourrait cependant imaginer, pour les installations travaillant en déversoir au fil de l'eau, une technique de construction basée sur la plus grande puissance au moindre investissement. Notamment certaines usines installées très audessous d'un grand fleuve on d'un lac ne prennent qu'une faible partie du débit et ont une hauteur de chute pratiquement constante. Dans la construction des turbines, il peut alor's paraître avantageux de subordonner le rendement qui peut ici, en somme, être quelconque à l'économie du coût de construction, en partictulier à la simplification du profil d'aube $\left(^{\star}\right)$.

\section{A. - Cas où l'eau est surabondante}

C'est en principe le cas des usines au fil de l'eau. Nous avons vu que dans ce cas le rendement technique n'a pas d'importance, puisque la quantité d'eau consommée ne compte pas. Ce qui compte, c'est la quantité d'énergie fournie au réseau et qui sera diminuée du fait de l'usure de la roue. A quelle variable peut-on rattacher le degré d'usure de la roue? En général, ce degré d'usure semble être surtout une fonction du débil cumulé turbiné par la roue. On peut admettre qu'il est seulement fonction du temps lorsque la hauteur de chute varie peu et que la turbine est constamment utilisée au maximum. Ce qui est une condition suffisamment réalisée en fait : en effet, dans une installation au fil de l'eau, on se contente souvent, pour s'adapter au régime du fleuve, de modifier le nombre des turbines en marche sans faire varier leur régime, lequel est ainsi maintenu au maximum sauf pour l'une d'elles qu'on règle, en période de basses eaux, de manière à utiliser tout le flux d'eau disponible sans faire baisser la hauteur de la retenue maintenue de manière à obtenir la puissance maximum des autres turbines.

En définitive, nous prendrons pour variable rattachée au degré d'usure une quantité $s$ (service de la turbine) qui pourra être, suivant les cas, soit le temps, soit le débit cumulé lequel n'a pas besoin d'être connu avec précision, soit encore, ce qui parait le plus simple, l'énergie cumuléc foumie, lorsque celle-ci est suffisamment proportionnelle au débit cumulé, c'est-à-dire lors-

(*) Cette simplifiention du tracé des profils parait pouvoir être conçue dans ce cas, non seulement en vue d'une réduction de la dépense de façonnage, mais aussi, éventuellement, on vue d'une réduction de la ritesse d'usure, du fait d'un moindre frottement de l'eau sur les aubes. 
que le régime de la rivière est tel que la hauteur de chute soit suffisamment constante (abstraction faite de très courtes périodes de grosse crue ou d'étiage).

\section{Calcul DE la PERTE}

Si l'on appelle $\mathrm{U}_{0}$ l'énergie fournie initialement par la turbine pour un accroissement unitaire de la variable $s, s$ étant la variable indépendante choisie (temps de fonctionnement écoulé, ou débit cumulé ou encore énergie cumulée fournie), la perte instantanée d'énergie, pour une valeur s de la variable, sera représentée par :

$$
d \mathrm{E}=\mathrm{U}_{0} \propto\left(s_{1}\right) d s
$$

$\alpha$ étant une fonction croissante de $s$ représentant la perte de puissance due à l'usure.
La valeur en francs de cette perte instantane d'énergie sera :

$$
d \pi=p \mathrm{U}_{0} \alpha(s) d s
$$

$p$ étant le prix de vente du $\mathrm{kWh}$ aux services de distribution.

Cette perte est a priori imputable à trois causes :

1. Le vieillissement de la roue;

2. Lc vieillissement de la conduite d'amenée;

3. Le vieillissement de l'alternateur.

Mais dans le laps de temps de l'usure de la roue, les causes (2) et (3) sont négligeables vis$\grave{a}$-vis de (1).

\section{B. - Cas où l'eau est mise en réserve}

Ici, chaque mètre cube d'eau stockée représente une énergie qu'il importe de ne pas gaspiller. La puissance fournie n'est pas en général la puissance maximum que peut fournir l'installation. Une usine à réservoir journalier peut avoir à fournir les pointes el à assurer la dentelle du diagramme à la demande du réseau. Le débit doit donc s'adapter très rapidement à la demande de puissance du réseau et à la hauteur de chute de manière à maintenir une tension sensiblement constante aux bornes de l'alternateur. Cette adaptation se fait par la manœuvre automatique du distributeur d'eau dont le servomoteur est commandé par de faibles variations de tension électrique. La puissance fournie demeure donc la même que si la roue ne s'usait pas, mais le rendement de celle-ci diminuant, la consommation d'eau augmente, et c'est cetie augmentation de consommation à valeur clonnce de la puissance demandée qui représente la perte encourue du fait de l'usure des aubes. Le calcul ne sera donc pas, en principe, le même que dans le cas $A$.

D'autre'part, la hauteur de chute étant ici variable, il n'y a pas en général corrélation entre le temps ou le débit cumulé et l'énergie fournie. Cependant nous remarquerons que si l'usine est placée très bas, cette variation de hautenr peut n'ètre qu'une faible fraction de la hauteur de chute, en sorte que la variation relative iemeure faible, plus faible même souvent que porr une usine au fil de l'eau où le niveatu aval peut va- rier beaucoup, la hauteur de chute allant même jusqu'à s'annuler en cas de crue.

Nous admettrons donc (il ne s'agit que d'un dégrossissage du problème) que la hauteur de chute est constante et égale à la moyenne des hauteurs réelles; la perte d'énergie pourra alors, ici encore, être considérée comme exclusivement fonction de la variable $s$, par l'intermédiaire, cette fois, du rendement technique.

\section{Calcul de la perte:}

La perte de rendement pour la valeur $s_{1}$ de la variable sera représentée par $\beta(s), \beta$ étant une fonction croissante de $\left(s_{1}\right)$.

La perte d'énergie instantanée est :

$$
d \mathrm{E}=\mathrm{U}_{0} \beta(s) d s
$$

Cette formule ne diffère de celle du cas A que par la substitution de la fonction $\beta$ à la fonction $\alpha$.

On en déduira la perte instantanée en francs :

$$
d \pi=p \mathrm{U}_{0} \beta(s) d s
$$

Tandis que dans le cas $A, \approx$ représentait un coefficient de chute de puissance, dans le cas $B$, $\beta$ représente un coefficient de chute de rendement. 


\section{C. - Recherche de l'optimum}

Nous voyons que dans les cas $A$ et $B$, l'expression de la perte élémentaire d'énergie due à l'usure de la roue a la même forme analytique a la signification près du coefficient de perte :

Premier cas A :

$$
d \pi=p \mathrm{U}_{0} \propto(s) d s
$$

Deuxième cas B :

$$
d \pi=p \mathrm{U}_{0} \beta(s) d s
$$

Nous continuerons le calcul avec la formule :

$$
d \pi=p U_{0} \alpha(s) d s
$$

étant entendu que, dans le cas $B, \approx$ doit ètre remplacé par $\beta$.

La perte $d \pi$ est évidemment d'autant plus petite que le remplacement est plus fréquent. Mais cet avantage du remplacement fréquent est compensé par les frais et sujétion dudit remplacement.

Soit $\Delta$ le service total de l'exploitation considéré comme donné et $\mathrm{N}$ le nombre de remplacements, que nous supposerons être un nombre entier assez grand. Si on désigne par $\mathrm{S}$ le service accompli par ehaque roue, entre sa pose et son remplacement, on a :

$$
\Delta=\mathbf{N} \mathrm{S}
$$

La perte est une fonction croissante de $S$, la dépense totale de remplacement est proportionnelle à $N=\Delta / S$.

Il s'agit de déterminer $\mathrm{S}$ de telle sorte que le total de la dépense de remplacement et de la perte en kWh soit minimum.

Pour une roue maintenue en exploitation jusqu'au service $S$, la dépense comprend :

- Des frais d'exploitation (surveillance, graissage), qu'on peut supposer proportionnels au temps, qui sont en tout cas indépendants de l'état d'usure de la roue; il n'y a pas à en tenir compte;

-- La dépense d'achat de la roue $E$;

- La dépense de dépose et de repose $F$.

Dans le cas $A$, il faut y ajouter la valeur $G$ du nombre de $\mathrm{kWh}$ non produits pendant le temps d'immobilisation de l'installation du fait du remplacement de la roue.
Pour calculer la dépense globale afférente à la roue, il faudrait, en toute rigueur, tenir compte du fait que, en raison de l'intérêt de l'argent, une dépense faite à une époque n'esl pas équivalente à la même dépense, faite à une autre époque. En fait, si la durée de service de Ja roue est assez faible (ce qu'on peut admettre, même pour les turbines à service intermittent), on peut négliger la considération des intérêts.

La dépense totale (y compris les pertes) afférente à la roue est alors supposée faite à un moment quelconque du temps de service de la roue. et elle est égale à la somme des dépenses élémentaires :

$$
\mathrm{D}=\mathrm{E}+\mathrm{F}+\mathrm{G}
$$

Pour le calcul, nous supposerons linéaire la loi d'usure $x_{(s)}$ qui devient $\alpha \mathrm{S}$; on trouve alors pour expression de $D$, en posant, pour simplifier lécriture, $\mathrm{E}+\mathrm{F}+\mathrm{G}=b$ et $p \mathrm{U}_{0} \alpha=\mathrm{K}$ :

$$
\mathrm{D}=b+\mathrm{K} \int_{0}^{\mathrm{s}} s d s=b+\frac{\mathrm{K} \mathrm{S}^{2}}{2}
$$

étant entendu que $\mathrm{S}$ représente, suivant la variable adoptée, soit la durée du service de la roue, soit la quantité d'eau turbinée par elle pendant cette durée, soit la quantité d'énergic correspondante; quelle que soit la signification attribuée à cette quantité $S$, nous la désignerons par le mot «période». Du moment que nous faisons abstraction des intérèts, nous pouvons aussi bien considérer cette dépense $D$ comme uniformément répartie le long de la période, avec une valeur moyenne par unité de la variable $s$, égale à :

$$
\hat{\mathrm{o}}=\frac{\mathrm{D}}{\mathrm{S}}=\frac{b}{\mathrm{~S}}+\frac{\mathrm{K} \mathrm{S}}{2}
$$

Le régime de remplacement optimum est celui qui correspond au minimum de $\hat{o}$. On peut expliquer ce fait, assez intuitif, en considérant que $\delta$ n'est autre que le coût moyen par unité de service fait. On peut aussi, plus explicitement, raisonner comme suit : soit, à une époque quelconque de l'exploitation de l'installation, $\Sigma$ une quantité de service fait quelque peu supérieure à la période de renouvellement. Si cette période est $S^{\prime}$, le coût sera :

$$
D^{\prime}=\delta^{\prime} S^{\prime}+\delta^{\prime}\left(\Sigma-S^{\prime}\right)=\delta^{\prime} \Sigma .
$$

Si la période est $S^{\prime \prime}$, le coût sera :

$$
D^{\prime \prime}=\delta^{\prime \prime} \mathrm{S}^{\prime \prime}+\delta^{\prime \prime}\left(\Sigma-\mathrm{S}^{\prime \prime}\right)=\delta^{\prime \prime} \Sigma
$$


supérieur ou inférieur au précédent, selon que $\delta^{\prime \prime} \gtrless \delta^{\prime}$.

Le minimum correspond bien au minimum de o donné immédiatement par l'équation :

$$
\frac{d \hat{a}}{d S}=-\frac{b}{S_{0}^{2}}+\frac{K}{2}=0
$$

où $\mathrm{S}_{0}=\sqrt{\frac{2 b}{\mathrm{~K}}}$ avec $\mathrm{K}=p \mathrm{U}_{0} \alpha$ ou $p \mathrm{U}_{0} \beta$, selon le cas.

Lorsque la variable s désigne le temps, la formule ci-dessus donne immédiatement les époques de remplacement des roues. Pour le cas où la variable désigne le débit turbiné, nous savons que des études très soignnées ont été faites indiquant pour les usines un programme rationncl de turbinage dans le temps. C'est à l'aide de ce programme que l'on pourrait préciser les époques de remplacement des roues.

Si au lieu de remplacer la roue tous les $S_{0}$ on la remplace seulement en moyenne tous les $S_{1}>S_{0}$ (le remplacement d'une roue trop tôt scmble peu probable hors du cas daccident), on a une perte moyenne d'argent par accroissement unitaire de la variable $s$ égale à :

$$
\begin{aligned}
& \frac{\frac{K}{2} S_{1}^{2}+b-\frac{K}{2} S_{0}^{2}+b}{S_{1}}-\frac{S_{0}}{2}-\left(S_{1}-S_{13}\right)\left(\frac{K}{2}-\frac{b}{S_{1} S_{0}}\right) \\
& \quad
\end{aligned}
$$

Si l'on se trompe de $100 \mathrm{n} \%$ sur la période de remplacement, on a :

$$
\mathrm{S}_{1}=\mathrm{S}_{\mathrm{p}}(1+n)
$$

et la perte unitaire est :

$$
n \mathrm{~S}_{0}\left\lceil\frac{p \mathrm{U}_{0} x}{2}-\frac{b}{(1+n) \mathrm{S}_{0}^{2}}\right\rceil
$$

cl la fraction du chiffre d'aftaires que représente celle perte est :

$$
\frac{n \mathrm{~S}_{0+}}{p \mathrm{U}_{0}}\left[\frac{p \mathrm{U}_{0} \alpha}{2}-\frac{b}{(1+n) \mathrm{S}_{0}^{2}}\right]
$$

\section{D. - Détermination des valeurs des $\alpha$ et $\beta$}

Nous ne savons pas si les fonctions de décroissance de puissance $\alpha$ et do rendement $\beta$ du fait de l'usure de la roue ont déjà fait l'objet de mesures précises. Il serait utile, s'il existe à tout le moins une loi moyenne, de connaître la courbe de cette loi.

Le coefficient $\alpha$ est un coefficient de réduction de puissance. Pour le déterminer en première approximation, on pourra par exemple procéder comme il suit :

On mesurera la puissance électricque initiale dans certaines conditions de marche: niveau amont, niveau aval, On débitera sur des résistances liquides (car la consommation du réseau est trop variable pour permettre une mesure). C'est la mesure de $U_{0}$ à l'époque $t_{0}$ et pour la valeur $s_{0}$ de la variable indépendante.

A l'époque $t_{1}$ de fonctionnement on s'efforcera d'avoir les mêmes niveaux amont et aval ou bien l'on fera si possible les corrections nécessaires. On obtiendra une nouvelle mesure $\mathrm{U}_{1}$ :

$$
\text { De : } \quad \begin{aligned}
\mathrm{U}_{1} & =\mathrm{U}_{0}(1-\alpha s) \\
& \text { on tire } \alpha .
\end{aligned}
$$

La détermination $d u$ coefficient $\beta$ est plus délicate. Il s'agit d'un coefficient de baisse du rendement technique.
En appelant $P$ la puissance électrique aux bornes de l'alternateur, $q$ le débit instantane, ? le rendement de la roue, $\theta$ le rendement de l'alternateur, $H$ la hauteur de chute nette, on a, au début de l'installation d'une roue.

$$
P_{0}=H q_{0}: 0
$$

A un aulre moment, en admethant constant le rendement de l'alternateur, la puissance pour la même hauteur de chute $H$ sera :

d'oil :

$$
\mathrm{P}_{1}=\mathrm{H} q_{1} p_{1} 0
$$

$$
P_{1}=-P_{0} \frac{q_{1}}{q_{0}} \frac{q_{1}}{p_{0}}
$$

mais $?_{1}=p_{0}(1-\beta S)$, de sorte qu'on a :

$$
P_{1}=P_{0} \frac{q_{1}}{q_{0}}(1-\beta S)
$$

équation d'où on tirera $\beta, S$ ćtant l'accroissement de la variable indépendante dans l'intervalle $t_{1}-t_{0}$.

Il ne suffit donc pas de faire les mesures électriques de $P_{0}$ et de $P_{1}$, il faut aussi mesurer $q_{0}$ et $q_{1}$. Ce sont des mesures de déhit, plus difficiles et moins précises (on admet $\pm 2 \%$ d'er- 
reur maximum). Coest une objection qui semble importante pour l'utilisation des calculs, car l'erreur est de l'ordre de la différence des quantités a mesurer. On peut imaginer que des mesures soignées en laboratoire sur modètes réduits puissent donner de bons résultats grâce à la propriété des turbines semblables d'avoir le même rendement. La difficullé serait d'obtenir la similitude de l'usure mais elle ne paraît pas insurmontable puisqu'on fait des itudes d'irosion sur modìles réduits.

Cependant, il serait beaucoup plus élegatut d'éviter toute mesure de débit et la chose paraît possible à l'aide d'une itude de corrélation. En effet, une turbine étant donnce et fonctionnant sous une hauteur nette $H$, on fait débiter l'alternateur sur une résistance liquide bien connuc. La turbine tournant à sa vitesse de régine automatiquement réglée absorbe un débit $q_{0}$ sous une ouverture $\omega_{0}$, l'alternateur fournissant une puissance $\mathrm{P}_{0,0}$. Or la vitesse étant constante, il y a corrélation simple entre le débit et l'ouverture w sous une hauteur nette donnée de sorte qu'il existe une courbe ouverlure-débil caractéristique de la turbine. Un groupe de lelles courbes pour différentes valeurs de $H$ paraît même pouvoir être tracé au moins en première approximation à l'étude de la turbine, de sorte que la simple lecture de louverture (position du distributeur) donnerait le débit comme une lecture sur les appareils électriques donne la puissance fournie.

$P_{0}$ et $P_{1}, q_{n}$ et $q_{1}$ ctant lus pour des valeurs de la variable indépendante différent de $\mathrm{S}$, la formule (4) donne $\beta$.

L'application du procédé peut permeltre, par la multiplicité des mesures, des observations de nature à préciser les courbes $q=f(\omega)$ et $\beta=g(s)$.

Cette dernière relation résoud le problème entièrement puisqu'un simple calcul donne ensuile la période optima de remplacement.

On peut encore, comme nous l'avons déjà dit, calculer pour une hauteur nette donnéc, et une résistance liquide donnée, la paissance à la fin d'une période optima et remplacer la roue quand cette puissance est alteinte.

\section{E. - Comparaison des valeurs de $x$ et $\beta$}

Supposons qu'on prenne le temps pour variable et qu'on fasse des mesures $\mathrm{P}_{0}$ et $\mathrm{P}_{1}, q_{0}$ et $q_{1}$ à un an d'intervalle $(t=1)$. On mesure, par exemple :

$$
P_{1}=0,98 P_{0}
$$

La formule (3) (cas A) donne :

$$
0,98 \mathrm{P}_{\mathrm{o}}=\mathrm{P}_{0}(1-\alpha) \text { d'où } \alpha=0,02 .
$$

Or, supposons qu'entre les deux mesures, et du fait de l'usure et des tourbillons, le débit instantané ait diminué de telle façon que $q_{1} / q_{0}=0,99$.

La formule (2) (cas B) donne alors :

$$
0,98 P_{0}=P_{0} \times 0,99(1-\beta S),
$$

ou, si l'on prend pour unite de S la quantite d'eau turbinée dans l'année:

$$
0,98 \mathrm{P}_{0}=\mathrm{P}_{0} \times 0,99(1-\beta)
$$

$$
\text { d'oủ : } \quad \beta=1-\frac{0,98}{0,99}=0,01
$$

L'écart entre $\beta$ et $\alpha$ est du simple au double et la période de remplacement calculée sera très différente. Les deux coefficients ne sont donc pas du tout équivalents.

Aussi la formule (1) convient-elle aux usines où l'eau est gratuite, tandis que la formule (2) convient aux usines à réservoir ou l'eau stockée equivaut à l'énergie en réserve.

\section{F. - Application numérique}

Nous prendrons une turbine du barrage de Rivières-sur-Tarn qui est une installation au fil de l'eau. C'est une turbine Kaplan $(\mathrm{H}=17 \mathrm{~m})$, la vitesse relative de l'eau est élevée. Cette turbine a une puissance de $11.000 \mathrm{~kW}$ et produit en année moyenne $(4.100 \mathrm{~h})$ une énergie de $45 \mathrm{mil}$ lions de $\mathrm{kWh}$.
Nous admettrons, pour fixer les idées, une chute de puissance de $1 \%$ au bout d'un an, chiffre qui paraît modeste. On a donc:

$$
\alpha=0,01
$$

On admettra un prix de vente du kWh de 
5 francs aux bornes de l'usine hydro-électrique et pour prix d'achat d'une roue neuve $510.000 \mathrm{fr}$. (roue usée déduite). Ce sont des ordres de grandeur de 1952.

\section{$1^{\circ}$ Calcul du coefficient $\mathrm{K}$ :}

$K=p P_{0} \alpha=5 \times 45 \times 10^{6} \times 0,01=2,25.10^{\circ} \mathrm{fr}$.

$2^{\circ}$ Cout $b$ de Remplacement d'une houe:

On admettra trente heures d'arrêt, soit :

$$
\frac{5 \times 45 \times 10^{6} \times 30}{4.100}=1,65.10^{\mathrm{i}} \mathrm{fr}
$$

soit au total, pour l'achat et la mise en place:

$$
b=510.000+1.650 .000=2,16.10^{6} \mathrm{fr} .
$$

$3^{\circ}$ On trouve alors, pour période oplima de remplacement de la roue :

$$
\mathrm{T}_{0}=\sqrt{\frac{2 \times 2,16 \cdot 10^{16}}{2,25 \cdot 10^{i}}}=\sqrt{1,92}=1,38
$$

soit seize mois environ.

4" Supposons quion commette une crreur de $50 \%$ en moyenne sur la période de remplacement. Ce chiffre paraît mềme optimiste si l'on a effectivement omis de calculer cette période.

Il en résulte une perte par an de:

$$
\begin{aligned}
& \left(S_{1}-S_{0}\right)\left[\frac{K}{2}-\frac{b}{S_{1} S_{0}}\right] \\
& \left.=0,5 \times 1,38 \mid \frac{2,25.10^{\circ}}{2}-\frac{2,16.10^{\prime \prime}}{1,5.1,38^{\prime}}\right\rceil \\
& =254.000 \mathrm{fr} .
\end{aligned}
$$

L'énergie produite représente :

$$
5 \times 45.10^{6}=225 \text { millions de francs, }
$$

d'où la perte en $\%$ du chiffre d'afraires :

$$
\frac{0,254}{225}=0,113 \%
$$

Avec une erreur de $100 \%$ sur la période de remplacement, on aurait une perte de :

$1,38\left\lceil\frac{2,25 \cdot 10^{6}}{2}-\frac{2,16 \cdot 10^{6}}{2.1,38}\right\rceil=770.000 \mathrm{fr}$. par : an

soit : $0,77 / 225=0,34 \%$ du chifre d'affaires, ce qui est important.

Si $x=0,02$ au lieu de 0,01 avec une erreur de $100 \%$ sur la période de remplacement, la perte représente une fraction du chiffre d'affaires de :

$$
\frac{1,38}{225.10^{4}}\left|\frac{2,25.10^{4} \times 2}{2} \frac{2,16.10^{i}}{2 \times 1,38}\right|=1,03 \%
$$

Ces chifres intéressent an prenter chef le producleur d'álectriciti.

Pour lecenomie générale du pays, il s'agit d'une perte de kWh qu'il aurait fill avoir en plus. Nous renons d'apprécier te gain financier, mais en ceonomite relle, la valeur des $k$ Wh perdus paraît supérieure à ees chifrres purement commerciaux que nous avons essayé d'etablir.

En effet, non seulement le cout de la fabricattion des roues utilisées en plus grand nombre peut se trouver abaisse, mais surtoul les kilowatts récupérés procurent un gain technique qui fait boule de neige, ces kilowatts ćlant aussitôt employés à la production. On augmente la puissance productrice du pays. Cela est qualitativement bien supérieur au gain financier réalisci sur la vente des lilowatts, surtout à une époque de pénurie d'énergic. 